\title{
Correlation of maternal neutrophil to lymphocyte ratio with fetal birth weight in preeclampsia
}

\author{
Zaima Ali ${ }^{1, *}$, Saima Zaki², Uzma Zafar ${ }^{3}$, Ambreen Tauseef ${ }^{4}$, Sundus Tariq ${ }^{5}$, Attiqa Khalid ${ }^{3}$ \\ ${ }^{\prime}$ Dept. of Physiology, Lahore Medical and Dental College Lahore/ University of Health Sciences Lahore, Pakistan \\ ${ }^{2}$ Dept. of Obstetrics and Gynecology, Jinnah Hospital Lahore, Pakistan \\ ${ }^{3}$ Dept. of Physiology, Lahore Medical and Dental College Lahore/ University of Health Sciences Lahore, Pakistan \\ ${ }^{4}$ Dept. of Physiology, CMH Lahore Medical College \& IOD/University of Health Sciences Lahore, Pakistan \\ ${ }^{5}$ Dept. of Physiology, University Medical and Dental College, University of Faisalabad/ University of Health Sciences \\ Lahore, Pakistan \\ *Corresponding Author: zaima.ali@hotmail.com
}

\begin{abstract}
Preeclampsia is a generalized inflammatory disorder complicating gestation, with most of the cases developing in the third trimester. To evaluate the neutrophil to lymphocyte ratio in diagnosed cases of preeclampsia and determine its correlation with fetal birth weight, a cross sectional analytical study was performed in Shaikh Zayed Medical Complex and Jinnah Hospital, Lahore. The participants included 60 cases of preeclampsia and 60 normotensive controls in the third trimester of pregnancy. All the participants were in the age group of 20-40 years and body mass index of 18-25. Total and differential leukocyte count was checked by automated hemoanalyzer, and the neutrophil lymphocyte ratio was calculated. Fetal birth weight was recorded immediately after birth. Statistical analysis was performed by SPSS (version 20). The $p$ value of $<0.05$ was considered significant. Neutrophil to lymphocyte ratio was significantly high ( $p$ value $<0.001)$ in the preeclamptic group with a median value of $2.64(2.0-3.8)$ as compared to $1.87(1.7-2.0)$ in the healthy group. The fetal birth weight was significantly low $(p$ value $<0.001)$ in the pregnancies complicated with preeclampsia. High neutrophil to lymphocyte ratio was negatively correlated with fetal birth weight in preeclampsia ( $p$ value $<0.01$ ). On logistic regression significant association of high neutrophil to lymphocyte ratio with low fetal birth weight was found after controlling for the confounder gestational age, with an odds ratio of 8.28 (95\% confidence interval 2.84-24.17). The area under the receiver operating characteristic curve for neutrophil to lymphocyte ratio was 0.8. The optimum cutoff value of neutrophil to lymphocyte ratio was 2.3 with $79 \%$ sensitivity and $76 \%$ specificity. High maternal neutrophil to lymphocyte ratio in the third trimester in preeclampsia negatively correlates with fetal birth weight and is a simple promising biomarker to predict low fetal birth weight.
\end{abstract}

Keywords: Fetal birth weight; lymphocytes; neutrophils; preeclampsia; pregnancy.

\section{Introduction}

Preeclampsia (PE) is a complication of the second or third trimester of pregnancy. It affects approximately 7 $-10 \%$ of pregnant women (Osungbade \& Ige, 2011). The disease contributes a large share to the maternal and fetal mortality. Abnormal placental development with incomplete remodeling of the spiral arteries results in hypoxia and release of proinflammatory cytokines. Oxidative stress and increased amount of toxins initiate widespread systemic inflammation (Aouache et al., 2018). Multiple disturbances in the hemostatic system such as the endothelial cell dysfunction, platelet activation, and enhanced intravascular coagulation evolve in the course of the disease (Kohli et al., 2016).

Disruption of the immune system has an important role in the pathophysiology of preeclampsia. In normal pregnancy, there is altered innate immune response with an intense inflammatory state and complement activation (Derzsy et al., 2010). This response is further accentuated in pregnancies complicated with preeclampsia (Denny et al., 2013). Elevated neutrophil counts with activation and excessive release of granule product elastase into maternal circulation and placental bed have been observed 
in preeclampsia (Catarino et al., 2012). Activation and modulation of neutrophils with increased production of superoxide and decrease in nitrite release have been attributed to widespread endothelial dysfunction in preeclampsia (Matsubara et al., 2015).

A number of inflammatory markers have been studied to measure systemic inflammation, related with severity of preeclampsia, e.g., $\mathrm{C}$ reactive protein (CRP), tumor necrosis factor $\alpha$ (TNF $\alpha$ ), and interleukin -6 (IL-6). Recently, hematological parameters including blood cell subtype ratios, such as neutrophil to lymphocyte (NLR) and platelet to lymphocyte (PLR) ratios, have been used as a prognostic and diagnostic tool for chronic diseases related to low-grade systemic inflammation (Kirbas et al., 2014; Arbel et al., 2012; Guthrie et al., 2013). Evidence of systemic inflammation in preeclampsia evoked the idea of comparing these ratios in preeclampsia and normal pregnancy (Ak1l et al., 2015) and predicting the severity of the disease (Toptas et al., 2016). Uteroplacental insufficiency compromises the blood flow to the fetus with resultant intrauterine growth retardation (IUGR). This effect on fetal growth is even more pronounced in severe preeclampsia resulting in $12 \%$ lower birth weight than expected (Backes et al., 2011). NLR is a readily available, simple, and inexpensive marker, which can be used as a powerful predictor of low fetal birth weight and for risk stratification in preeclampsia. We hypothesized that increased maternal NLR in preeclampsia correlates with low fetal birth weight. In the present study, we calculated maternal NLR, recorded fetal birth weight, and determined the correlation of maternal NLR in the third trimester with fetal birth weight in preeclamptic and normal pregnancies.

\section{Materials \& methods}

This cross-sectional analytical study was approved by the Ethical Review Boards of Shaikh Zayed Medical Complex and Jinnah Hospital, Lahore. The study was conducted from February 2012 till August 2012. Study population consisted of 60 normotensive and 60 preeclamptic pregnant women (aged 20-40 years) in the third trimester of pregnancy. The sample size was obtained by using 5\% level of significance and $80 \%$ power of test with expected fetal birth weight of $3.0 \pm 0.49$ and $3.26 \pm 0.40 \mathrm{Kg}$ for preeclamptic and normotensive groups respectively (Lau et al., 2005).

Preeclampsia (PE) was diagnosed as onset of systolic blood pressure $>140 \mathrm{mmHg}$ or diastolic blood pressure $\geq 90 \mathrm{mmHg}$ at $>20$ week of gestation accompanied by 24 - hour proteinuria $\geq 300 \mathrm{mg}(\geq 1+$ on dipstick), in at least two random urine samples collected $4-6$ hours apart. Women with history of smoking, chronic hypertension, diabetes, arthritis, renal disease, inflammatory bowel disease, any cardiovascular illness, and symptomatic infectious disease (bacterial and viral) were excluded.

\subsection{Sample collection}

Blood pressure was recorded, and maternal venous blood samples were collected in EDTA coated vacutainer. Total leukocyte counts including neutrophils and lymphocytes were calculated by automated hemoanalyzer, and NLR was calculated. Fetal birth weight was recorded immediately after delivery.

The study followed all the ethical standards of the Institutional Ethical Review and the 1975 Helsinki Declaration revised in 2000. Informed written consent was obtained from all individual participants included in the study.

\subsection{Statistical analysis}

Statistical analysis was performed by using SPSS version 20. Normality of the data was checked by Shapiro Wilk test. Data was presented as median with quartile range as it was nonnormally distributed. Mann-Whitney U test was used for comparison between the two groups. Spearman correlation was used to check the correlation between quantitative variables. Receiver operating characteristic (ROC) curve was plotted to find the optimal cutoff value. Overall diagnostic accuracy was estimated with the area under the ROC curve. Binary logistic regression was used to assess the predictability of fetal birth weight on the basis of NLR.

\section{Results}

Data was recorded and divided into two groups, Group I and Group II, comprising of 60 preeclamptic and 60 normotensive pregnant women, respectively. Maternal age, gravidity, and parity were not different between the two groups. NLR was significantly high ( $p$ value $<$ 0.001 ) in the preeclamptic group with a median value of 2.64 (2.0-3.8) as compared to 1.87 (1.7-2.0) in the healthy group. The fetal birth weight was significantly low ( $p$ value $<0.001$ ) in the pregnancies complicated with preeclampsia (Table 1). Spearman correlation coefficient between NLR and fetal birth weight was -0.384 in the preeclamptic group $(p$ value $<0.01)$ showing moderate reverse relationship (Figure 1). 
Table 1. Clinical and hematologic characteristics of the participants.

\begin{tabular}{llll}
\hline Variable & $\begin{array}{l}\text { Preeclampsia } \\
\text { median (IQR) }\end{array}$ & $\begin{array}{l}\text { Normotensive } \\
\text { median (IQR) }\end{array}$ & $* p$ value \\
\hline Gravidity & $2(1-4)$ & $2(1-5)$ & 0.38 \\
Parity & $1(0-2)$ & $1(0-3)$ & 0.59 \\
Gestational age at birth (weeks) & $38(37.1-39)$ & $40(39-40)$ & $<0.001$ \\
SBP $(\mathrm{mmHg})$ & $150(140-150)$ & $110(110-120)$ & $<0.001$ \\
DBP $(\mathrm{mmHg})$ & $100(100-100)$ & $70(70-80)$ & $<0.001$ \\
TLC $\left(\times 10^{3} / \mathrm{cumm}\right)$ & $10.1(8.9-11.0)$ & $7.1(5.9-8.4)$ & $<0.001$ \\
NLR & $2.64(2.0-3.8)$ & $1.87(1.7-2.0)$ & $<0.001$ \\
FBW $(\mathrm{Kg})$ & $2.5(2.3-2.9)$ & $3.2(2.9-3.3)$ & $<0.001$ \\
\hline
\end{tabular}

Systolic Blood Pressure (SBP), Diastolic Blood Pressure (DBP), Total Leukocyte Count (TLC), Neutrophil to Lymphocyte ratio (NLR), FBW (Fetal Birth Weight), * $p$ value calculated by Mann-Whitney U test, $\mathrm{p}$ value $<0.05$ considered significant.

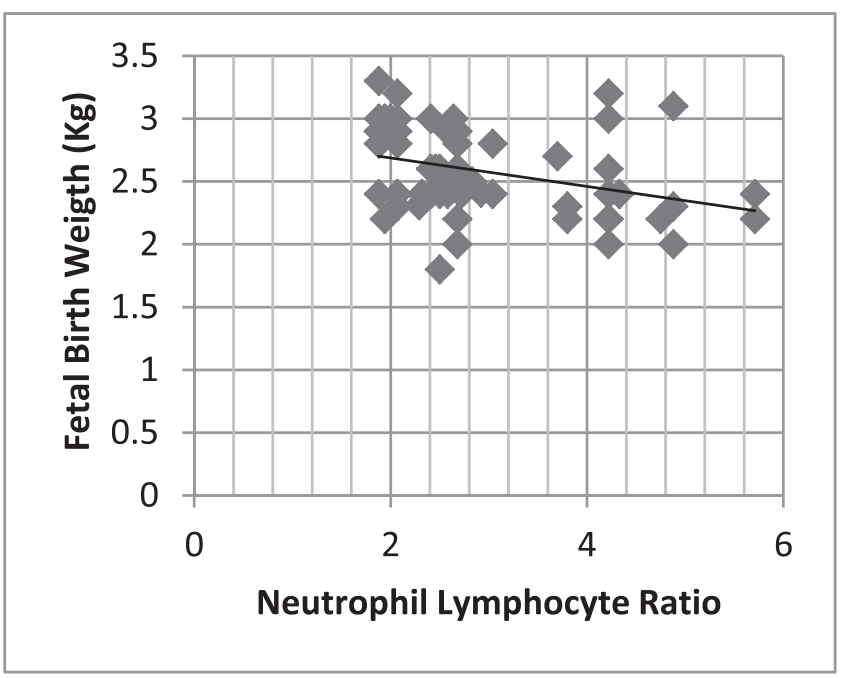

Fig. 1. Correlation of NLR with fetal birth weight in preeclampsia Spearman $(\rho)=-0.384, p<0.01$ (Spearman's rank correlation test showing moderate negative correlation between NLR and fetal birth weight).

Gestational age at birth was significantly different between the two groups $(p$ value $<0.01$ ). The partial correlation between NLR and fetal birth weight adjusted for fetal birth weight was -0.0232 ( $p$ value $<0.01)$. ROC curve analysis revealed a value of 0.800 for the area under the curve (AUC). The optimum cutoff value of NLR was 2.3 with $79 \%$ sensitivity, $76 \%$ specificity, and a false positive rate of $24 \%$. When the fetal birth weight was regressed on NLR and gestational age at birth in binary form, the predictability of birth weight on the basis of NLR was $75.8 \%$ with $100 \%$ normal weight prediction. Binary logistic regression calculated an odds ratio of 12.02 (4.34$33.2995 \% \mathrm{CI})$. After adjusting for gestational age, the accuracy in prediction of birth weight improved to $82.5 \%$ with underweight prediction accuracy of 34.5 and normal weight prediction of $97.8 \%$ (Table 2). Binary logistic regression with NLR as predictor and gestational age at birth as confounder showed an odds ratio of 8.28. This showed that when the effect of gestational age at birth is controlled, there is 8.28 times increased risk of low fetal birth weight with high levels of NLR in the third trimester of pregnancy. 
Table 2. Predictability of NLR for fetal birth weight.

\begin{tabular}{|c|c|c|c|}
\hline \multicolumn{4}{|c|}{ Predictability of NLR for fetal birth weight } \\
\hline & Predicted & & Percentage \\
\hline & Low birth weight & Normal + & Correct \\
\hline \multicolumn{4}{|l|}{ Observed } \\
\hline Low weight & 0 & 29 & 0 \\
\hline Normal & 0 & 91 & 100 \\
\hline Overall percentage & & & 75.8 \\
\hline \multicolumn{4}{|c|}{ Predictability of NLR for fetal birth weight keeping gestational age at birth as confounder } \\
\hline & Predicted & & Percentage \\
\hline & Low birth weight & Normal + & Correct \\
\hline \multicolumn{4}{|l|}{ Observed } \\
\hline Low weight & 10 & 19 & 34.5 \\
\hline Normal & 2 & 89 & 97.8 \\
\hline Overall percentage & & & 82.5 \\
\hline
\end{tabular}

\section{Discussion}

This study has reported significantly higher maternal NLR in the third trimester in preeclampsia as compared to healthy controls. In addition, it was observed that NLR negatively correlated with fetal birth weight. The results are congruous with a number of studies that support the role of insistent and enhanced systemic inflammation in the pathogenesis of endothelial dysfunction in preeclampsia (Pinheiro et al., 2013; Can et al., 2011; Deveci et al., 2009). The etiology of preeclampsia is multifactorial with the commonest concept being poor placental development leading to hypoxia and release of proinflammatory cytokines. There is evidence of immune maladaptation in preeclampsia (Denny et al., 2013).

Systemic inflammation leads to increased number of white blood cells. Neutrophils play a very important role and are the first blood cells to respond to inflammation. In recent past, elevated NLR has been studied as a marker of systemic inflammatory response in a number of clinical conditions, e.g., cardiovascular diseases, malignancies, and preeclampsia (Guthrie et al., 2013; Suliman et al., 2010; Kurt et al., 2015). The higher total leukocyte count in the preeclamptic pregnancies as compared to normotensive group reported here is in accordance with previous researches (Yavuzcan et al., 2014; Gogoi et al., 2019). Increased neutrophil count with relative static lymphocyte number results in increased NLR, used as a marker of systemic inflammatory response.
In healthy pregnancy there is a shift of immune response with predominance towards $\mathrm{T}$ helper 2 (Th2) type cytokine environment, important for the survival of the fetus. This shift does not occur in pregnancies complicated by preeclampsia, and the Th1 response is not suppressed with resultant production of type 1 cytokines, e.g., TNF - $\alpha$, IL-2, Interferon gamma and inflammation (Ribeiro et al., 2017; Molvarec et al., 2010). A significantly high NLR in preeclampsia as compared to the healthy group is in accordance with the previous reports (Ak1l et al., 2015; Toptas et al., 2016). Recently, high NLR has been reported as an independent biomarker to predict preeclampsia (Oylumlu et al., 2014) and as a promising marker in the detection of severity of the disease (Sachan et al., 2017).

In addition to high neutrophil to lymphocyte ratio, this study has observed a significant difference in the birth weight of babies born to preeclamptic mothers as compared to healthy women with a low birth weight in the diseased group. Incomplete transformation of uterine arteries in preeclampsia results in compromised blood flow to fetus and ischemia. This ischemia leads to development of intrauterine growth restriction and low fetal birth weight (Backes et al., 2011). Activation of peripheral blood neutrophils has been documented in both preeclampsia and IUGR (Oggé et al., 2010).

In the present study, we tested and proved the hypothesis that high maternal NLR in the third trimester is correlated 
with low fetal birth weight in preeclampsia in accordance with a recent research (Akgun et al., 2017). Albeit birth weight was significantly low in the diseased group, this could be attributed to the difference in gestational age at birth. Regression analysis was done to control the effect of gestational age on fetal birth weight, and the results revealed 8.2 times increased risk of having low fetal birth weight with high maternal NLR in the third trimester in preeclampsia. In the receiver operating characteristic analysis, the AUC has a value of 0.8 , indicating maternal NLR as a powerful predictor of low fetal birth weight. In contrast to most of the inflammatory biomarkers, NLR is readily available, simple and inexpensive marker that can be used for risk stratification in preeclampsia.

A single sample from each participant in the third trimester of pregnancy is the limitation of our study. Longitudinal studies with multiple samples in all the three trimesters could strengthen our results and usefulness of this simple readily available test.

\section{Conclusion}

This study reports significantly high NLR in preeclampsia as compared to normotensive pregnancy. Moreover, high maternal NLR in the third trimester in preeclampsia negatively correlates with fetal birth weight. To the best of our knowledge the correlation of high NLR with low fetal birth weight in preeclampsia is reported for the first time. NLR, a readily available hematologic biomarker, can be used to predict low fetal birth weight in pregnancies complicated with preeclampsia with a sensitivity and specificity of $79 \%$ and $76 \%$, respectively.

\section{ACKNOWLEDGEMENTS}

The authors are thankful to all our subjects and technical staff of hospitals for their cooperation.

\section{References}

Akgun, N., Namli Kalem, M., Yuce, E., Kalem, Z. \& Aktas, H. (2017). Correlations of maternal neutrophil to lymphocyte ratio (NLR) and platelet to lymphocyte ratio (PLR) with birth weight. J Matern Fetal Neonatal Med, 30(17):2086-2091.

Akıl, M.A., Bilik, M.Z., Acet, H., Tunç, S.Y., Ertaş, F., et al. (2015). Mean platelet volume and neutrophil lymphocyte ratio as new markers of preeclampsia severity. Kosuyolu Heart J, 18(2):84-88.

Aouache, R., Biquard, L., Vaiman, D. \& Miralles, F.
(2018). Oxidative stress in preeclampsia and placental diseases. Int J Mol Sci 19(5):1496.

Arbel, Y., Finkelstein, A., Halkin, A., Birati, E.Y., Revivo, M., et al. (2012). Neutrophil/lymphocyte ratio is related to the severity of coronary artery disease and clinical outcome in patients undergoing angiography. Atherosclerosis, 225(2):456-460.

Backes, C.H., Markham, K., Moorehead, P., Cordero, L., Nankervis, C.A., et al. (2011). Maternal preeclampsia and neonatal outcomes. Journal of pregnancy, 2011.

Can, M., Sancar, E., Harma, M., Guven, B., Mungan, G., et al. (2011). Inflammatory markers in preeclamptic patients. Clin Chem Lab Med, 49(9):1469-1472.

Catarino, C., Santos-Silva, A., Belo, L., Rocha-Pereira, P., Rocha, S., et al. (2012). Inflammatory disturbances in preeclampsia: relationship between maternal and umbilical cord blood. Journal of pregnancy, 2012:684384.

Denny, K.J., Woodruff, T.M., Taylor, S.M. \& Callaway, L.K. (2013). Complement in pregnancy: a delicate balance. Am J Reprod Immunol, 69(1):3-11.

Derzsy, Z., Prohászka, Z., Rigó Jr, J., Füst, G. \& Molvarec, A. (2010). Activation of the complement system in normal pregnancy and preeclampsia. Mol Immunol, 47(7-8):1500-1506.

Deveci, K., Sogut, E., Evliyaoglu, O. \& Duras, N. (2009). Pregnancy-associated plasma protein-A and C-reactive protein levels in pre-eclamptic and normotensive pregnant women at third trimester. J Obstet Gynaecol Res, 35(1):94-98.

Gogoi, P., Sinha, P., Gupta, B., Firmal, P. \& Rajaram, S. (2019). Neutrophil-to-lymphocyte ratio and platelet indices in pre-eclampsia. Int $\mathrm{J}$ Gynaecol Obstet, 144(1):16-20.

Guthrie, G.J., Charles, K.A., Roxburgh, C.S., Horgan, P.G., Mcmillan, D.C., et al. (2013). The systemic inflammation-based neutrophil-lymphocyte ratio: experience in patients with cancer. Crit Rev Oncol Hematol, 88(1):218-230.

Kirbas, A., Biberoglu, E., Daglar, K., İskender, C., Erkaya, S., et al. (2014). Neutrophil-to-lymphocyte ratio as a diagnostic marker of intrahepatic cholestasis of pregnancy. Eur J Obstet Gynecol Reprod Biol, 180(1215.

Kohli, S., Ranjan, S., Hoffmann, J., Kashif, M., Daniel, E.A., et al. (2016). Maternal extracellular vesicles 
and platelets promote preeclampsia via inflammasome activation in trophoblasts. Blood, 128(17):2153-2164.

Kurt, R.K., Aras, Z., Silfeler, D.B., Kunt, C., Islimye, M., et al. (2015). Relationship of red cell distribution width with the presence and severity of preeclampsia. Clin Appl Thromb Hemost, 21(2):128-131.

Lau, T.K., Pang, M.W., Sahota, D.S. \& Leung, T.N. (2005). Impact of hypertensive disorders of pregnancy at term on infant birth weight. Acta Obstet Gynecol Scand, 84(9):875-877.

Matsubara, K., Higaki, T., Matsubara, Y. \& Nawa, A. (2015). Nitric oxide and reactive oxygen species in the pathogenesis of preeclampsia. Int J Mol Sci, 16(3):46004614.

Molvarec, A., Ito, M., Shima, T., Yoneda, S., Toldi, G., et al. (2010). Decreased proportion of peripheral blood vascular endothelial growth factor-expressing $\mathrm{T}$ and natural killer cells in preeclampsia. Am J Obstet Gynecol, 203(6):567. e1-567. e8.

Oggé, G., Romero, R., Chaiworapongsa, T., Gervasi, M.T., Pacora, P., et al. (2010). Leukocytes of pregnant women with small-for-gestational age neonates have a different phenotypic and metabolic activity from those of women with preeclampsia. J Matern Fetal Neonatal Med, 23(6):476-487.

Osungbade, K.O. \& Ige, O.K. (2011). Public health perspectives of preeclampsia in developing countries: implication for health system strengthening. J Pregnancy, 2011.

Oylumlu, M., Ozler, A., Yildiz, A., Oylumlu, M., Acet, H., et al. (2014). New inflammatory markers in preeclampsia: echocardiographic epicardial fat thickness and neutrophil to lymphocyte ratio. Clin Exp Hypertens, 36(7):503-507.

Pinheiro, M.B., Martins-Filho, O.A., Mota, A.P.L., Alpoim, P.N., Godoi, L.C., et al. (2013). Severe preeclampsia goes along with a cytokine network disturbance towards a systemic inflammatory state. Cytokine, 62(1):165-173.

Ribeiro, V.R., Romao-Veiga, M., Romagnoli, G.G., Matias, M.L., Nunes, P.R., et al. (2017). Association between cytokine profile and transcription factors produced by T-cell subsets in early- and late-onset preeclampsia. Immunology, 152(1):163-173.

Sachan, R., Patel, M.L., Sachan, P. \& Shyam, R.
(2017). Diagnostic accuracy of neutrophil to lymphocyte ratio in prediction of nonsevere preeclampsia and severe preeclampsia. J Curr Res Sci Med, 3(2):79.

Suliman, M.A.R.M., Juma, A.A.B., Almadhani, A.A.A., Pathare, A.V., Alkindi, S.S.A., et al. (2010). Predictive value of neutrophil to lymphocyte ratio in outcomes of patients with acute coronary syndrome. Arch Med Res, 41(8):618-622.

Toptas, M., Asik, H., Kalyoncuoglu, M., Can, E. \& Can, M.M. (2016). Are Neutrophil/Lymphocyte Ratio and Platelet/Lymphocyte Ratio Predictors for Severity of Preeclampsia? Clin. Obstet. Gynaecol., 5(1):27-31.

Yavuzcan, A., Caglar, M., Ustun, Y., Dilbaz, S., Yidiz, E., et al. (2014). Mean platelet volume, neutrophillymphocyte ratio and platelet-lymphocyte ratio in severe preeclampsia. Ginekol Pol, 85(3).

Submitted : 06/12/2019

Revised : :17/02/2020

Accepted : 11/03/2020

DOI $\quad: 10.48129 / k j s . v 48 i 1.8769$ 